\title{
Short course acid suppressive treatment for patients with functional dyspepsia: results depend on Helicobacter pylori status
}

\author{
A L Blum, R Arnold, M Stolte, M Fischer, H R Koelz, and the Frosch Study Group $†$
}

\begin{abstract}
Background and aims-Treatment of functional dyspepsia with acid inhibitors is controversial and it is not known if the presence of Helicobacter pylori infection influences the response.

Methods-After a complete diagnostic workup, 792 patients with functional dyspepsia unresponsive to one week of low dose antacid treatment were randomised to two weeks of treatment with placebo, ranitidine $150 \mathrm{mg}$, omeprazole $10 \mathrm{mg}$, or omeprazole $20 \mathrm{mg}$ daily. Individual dyspeptic and other abdominal symptoms were evaluated before and after treatment
\end{abstract} according to $\mathrm{H}$ pylori status.

Results-The proportions of patients considered to be in remission (intention to treat) at the end of treatment with placebo, ranitidine $150 \mathrm{mg}$, omeprazole $10 \mathrm{mg}$, and omeprazole $20 \mathrm{mg}$ were, respectively, $42 \%, 50 \%, 48 \%$, and $59 \%$ in the $H$ pylori positive group and $66 \%, 73 \%$, $64 \%$, and $71 \%$ in the $H$ pylori negative group. In $H$ pylori positive patients, the therapeutic gain over placebo was significant for omeprazole $20 \mathrm{mg}(17.6 \%, 95 \%$ confidence intervals (CI) 4.2-31.0; $p<0.014$ using the Bonferroni-adjusted $p$ level of 0.017 ) but not for omeprazole $10 \mathrm{mg}$ $(6.8 \%, 95 \%$ CI $-6.7-20.4)$ or ranitidine 150 mg (8.9\%, 95\% CI -4.2-21.9). There was no significant therapeutic gain from active treatment over placebo in $H$ pylori negative patients. Complete disappearance of symptoms and improvement in quality of life also occurred most frequently with omeprazole $20 \mathrm{mg}$ and was significant in both $H$ pylori positive and $H$ pylori negative groups. The six month relapse rate of symptoms requiring treatment was low $(<20 \%)$ in all groups.

Conclusions-Omeprazole $20 \mathrm{mg}$ per day had a small but significant favourable effect on outcome in $H$ pylori positive patients. The differential response in these patients may be explained by an enhanced antisecretory response in the presence of $H$ pylori. The effect of weaker acid inhibition was unsatisfactory.

(Gut 2000;47:473-480)

Keywords: functional dyspepsia; omeprazole; ranitidine; Helicobacter pylori; randomised controlled trial; quality of life

There is still no adequate treatment for functional dyspepsia. In particular, the evi- dence for treatment of functional dyspepsia with acid pump blockers and other antisecretory agents is contradictory. Recently, two large controlled clinical trials conducted in parallel yielded conflicting results; in one trial, pump blockers appeared to be effective and in the other they were completely ineffective, although the protocols of the two trials were similar. ${ }^{1}$ In another study, only certain subgroups with a posteriori defined symptoms appeared to respond to omeprazole treatment. ${ }^{2}$ The question of whether omeprazole treatment is effective is all the more important as other treatment alternatives lack promise. ${ }^{3}$ There is increasing evidence, for example, that Helicobacter treatment of functional dyspepsia does not improve dyspeptic symptoms.

Therefore, we conducted a large controlled clinical trial in which we tested the effectiveness of two antisecretory agents, omeprazole and ranitidine. Ranitidine was given at the dose presently recommended for over the counter treatment. ${ }^{4}$ We intended to conduct the trial in a manner which most closely resembled routine clinical practice. Thus we chose as a main outcome criterion the disappearance of dyspeptic symptoms requiring further treatment, instead of complete disappearance of peptic symptoms. Additionally, we decided not to blind the investigators to Helicobacter pylori status. In Germany, where this trial was conducted, a large proportion of dyspeptic patients already know their $H$ pylori status. Excluding those patients would have led to selection bias. In addition, it is still common clinical practice to give antibiotics to those patients who have adequately responded to symptomatic treatment. ${ }^{5}$ We tested the validity of this attitude in a second trial including those patients who did not adequately respond in the present study. The results of that trial will be reported elsewhere. Thus in the present study the effectiveness of antisecretory treatment was assessed separately in $H$ pylori positive and negative subjects with functional dyspepsia. This is of particular interest as it has previously been shown that antisecretory agents, in particular proton pump inhibitors, are more effective in $H$ pylori positive than in $H$ pylori negative subjects. ${ }^{6}$

Patients and methods STUDY PROTOCOL

A multicentre, double blind, double dummy, randomised clinical trial with parallel groups was conducted according to Good Clinical

Abbreviations used in this paper: QoL, quality of life; ITT, intention to treat; PP, per protocol. 
Practice and the revised Declaration of Helsinki. The ethics committee at each centre approved the protocol and all patients gave written informed consent.

SELECTION OF PATIENTS

Recruitment took place between August 1994 and July 1996 and included 801 ambulatory patients of both sexes from 71 private gastroenterological practices in Germany, aged $\geqslant 18$ years, with chronic functional dyspepsia, with or without gastritis due to $H$ pylori. Chronic functional dyspepsia was defined as epigastric symptoms in the absence of organic disease known to produce epigastric symptoms. Initial symptoms had to be severe enough to require treatment. Dyspeptic symptoms necessitating treatment had to be present for at least four weeks and be severe on at least three days of the seven day run in screening period.

We excluded patients with heartburn/acid regurgitation without concomitant epigastric symptoms and also those with symptoms suggesting irritable bowel syndrome (pain in the lower abdomen, flatulence, diarrhoea, or constipation) which were severe enough to require treatment or diagnostic tests. In addition, patients with any of the following were excluded: current or previously documented erosive or ulcerative oesophagitis, peptic ulcer, or previous abdominal surgery (except for inguinal hernia, appendicectomy, hysterectomy, or Caesarean section); treatment with proton pump inhibitors and/or antibiotic treatment within one month prior to screening or regular treatment within the previous week with other drugs which might interfere with the study outcome; symptoms indicative of serious disease (for example, unintended weight loss, haematemesis) during the previous three months; and conditions associated with poor study compliance (for example, drug addiction or alcoholism).

\section{BASELINE INVESTIGATIONS}

At baseline, a complete medical history was taken followed by physical examination, abdominal sonography, and gastroscopy with biopsy (only axial hernia, less than 10 gastric erosions and endoscopic signs attributed to $H$ pylori induced gastritis were permitted at gastroscopy). Biopsy specimens were taken from the corpus (greater curvature) and from the antrum ( $3 \mathrm{~cm}$ proximal to the pylorus) for histological examination using the Sydney criteria $^{7}$ for assessment of $H$ pylori and gastritis. Rapid urease tests ${ }^{8}$ (HUT, Astra Chemicals, Wedel, Germany) were also performed and these were considered positive for $H$ pylori if at least one sample of the gastric corpus or antrum caused red discolouration within 24 hours. A validated ${ }^{13} \mathrm{C}$ urea breath test was also performed. ${ }^{8} \mathrm{H}$ pylori infection was diagnosed when, in addition to a positive HUT test, histological examination showed Helicobacterlike organisms in the corpus and/or antrum. The investigators were aware of the HUT test results but were blinded to the histological results throughout the study. Blood samples were taken at entry for routine assessment of haematology and clinical chemistry (one minor transitory abnormality was permitted) and these were repeated after the two week double blind treatment. Additional investigations, for example colonoscopy or CT scan, were done at the investigator's discretion if clinically justified and all findings had to be normal for inclusion in the study.

\section{STUDY TREATMENTS}

Patients were given antacid tablets which could be taken up to three times a day during the seven day run in screening period. These were Maaloxan tablets (Rhône-Poulenc-Rorer, Germany) which contained $200 \mathrm{mg}$ of aluminium hydroxide and $200 \mathrm{mg}$ of magnesium hydroxide, with an acid binding capacity of $11 \mathrm{mmol}$ $\mathrm{HCl}$ per tablet. Those patients who had severe dyspeptic symptoms on $\geqslant 3$ days during the run in screening period were eligible for study treatment. They were randomised and received omeprazole (Losec, Astra Pharmaceuticals Production AB, Sweden) capsules $10 \mathrm{mg}$ (OM10) or $20 \mathrm{mg}$ before breakfast (OM20), ranitidine (Zantac, Glaxo SpA, Italy) tablets $150 \mathrm{mg}$ in the evening before retiring, or placebo tablets and capsules (matched for appearance, taste, and smell) for 14 days. A visit with assessment of symptoms (see below) was carried out at the end of this treatment and if no further management was required (that is, medical treatment other than liquid antacids and/or diagnostic procedures such as endoscopy), patients entered a follow up period of up to six months. With their consent, $H$ pylori positive patients with symptoms requiring further treatment entered a second study evaluating $H$ pylori treatment (fig 1 ). For $H$ pylori negative patients with persistent symptoms at two weeks, the study was terminated, except for a personal visit or a telephone interview six months later.

\section{FOLLOW UP PERIOD}

Follow up of patients without symptoms requiring further treatment lasted for six months or until relapse of dyspepsia. During this period, no treatment for dyspepsia other than an antacid suspension $(10 \mathrm{ml}$ bags containing $3.13 \mathrm{~g}$ of aluminium hydroxide gel, $0.27 \mathrm{~g}$ of magnesium oxide, and $0.63 \mathrm{~g}$ of magnesium carbonate, with an acid binding capacity of $33 \mathrm{mmol} / \mathrm{ml}$ ) for occasional dyspeptic symptoms was allowed. Unscheduled visits were encouraged at any time during the six month follow up period when dyspeptic symptoms recurred, when antacid treatment was needed for more than four consecutive days, and/or when antacid consumption exceeded $30 \mathrm{ml}$ per day. Relapse was defined as reappearance of symptoms requiring management (treatment other than liquid antacid and/or diagnostic tests such as endoscopy).

OUTCOME CRITERIA

The main outcome criterion, tested at two weeks, was lack of dyspeptic symptoms requiring further management (as defined above). In addition, the severity of individual dyspeptic and other abdominal symptoms (specifically 


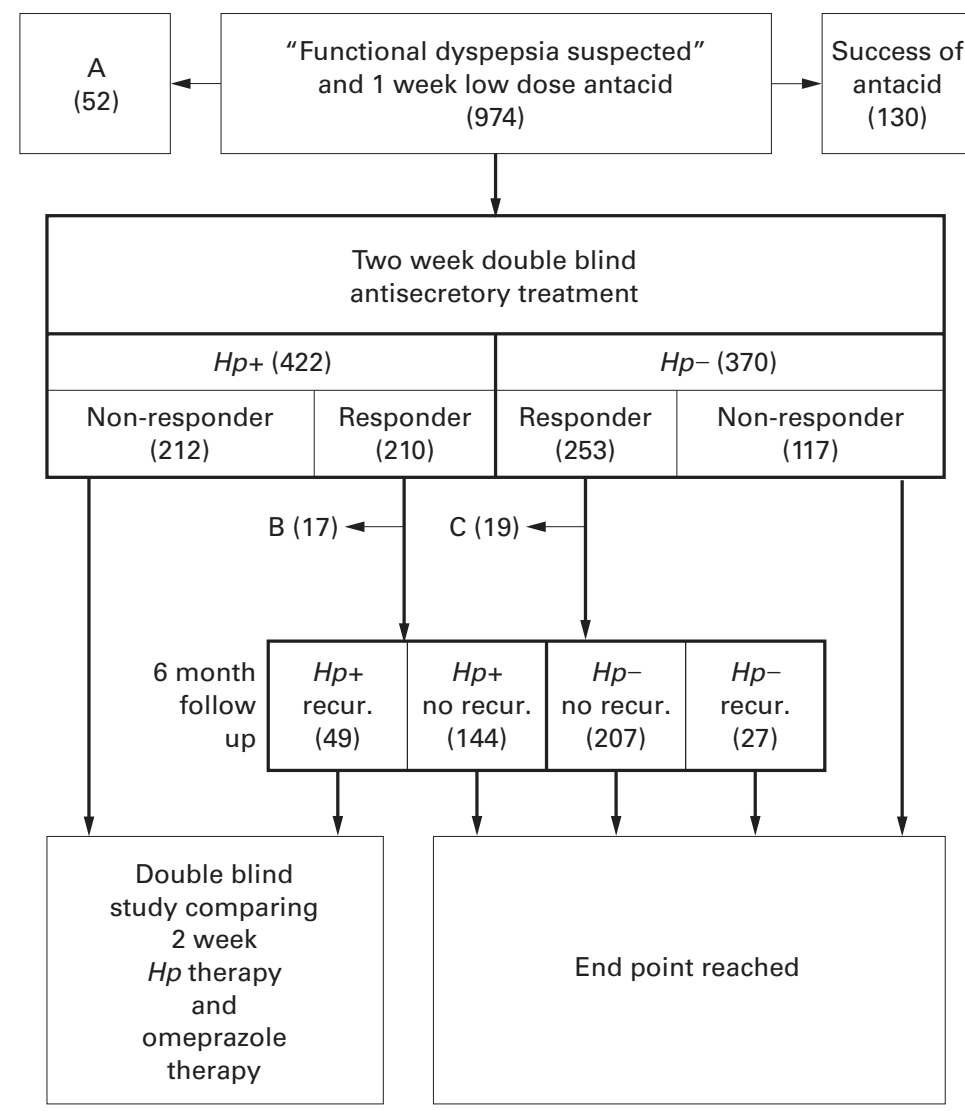

Figure 1 Flow chart of the 974 dyspeptic patients who entered the run in week with low dose antacid treatment. $A=$ not randomized after run in period for reasons other than response to antacid treatment $(n=52) . B=H$ pylori positive responders not entering follow up $(n=17) . C=H$ pylori negative responders not entering follow up $(n=19)$. Responder $=n o$ dyspeptic symptoms requiring further management after the two week double blind treatment (main outcome criterion). Hp, Helicobacter pylori; recur., recurrence of dyspeptic symptoms requiring management

epigastric pain/burning, epigastric pressure/ fullness, heartburn, acid regurgitation, nausea, vomiting, pain in the lower abdomen, flatulence, diarrhoea, and constipation) during the previous week was graded according to a four point scale $(0=$ no complaints, $1=$ complaints not interfering with daily activities and not requiring treatment, $2=$ complaints requiring treatment but not interfering with daily activities, and $3=$ complaints interfering with daily activities and requiring treatment). Patients answered the question "How were your symptoms in the area of the oesophagus and stomach" and "How was your general condition during the last seven days" by making a mark on a $10 \mathrm{~cm}$ visual analogue scale $(10 \mathrm{~cm}$, best possible condition; $0 \mathrm{~cm}$, worst condition). Quality of life (QoL) during the previous week was assessed using a validated questionnaire adapted to German lifestyles. ${ }^{9}$ It contained 40 general items relating to physical strength, ability to enjoy and relax, positive mood, absence of negative mood, social contacts, and social well being. In addition, nine questions validated in Germany, relating to impairment of QoL by dyspeptic symptoms, ${ }^{10}$ were asked. They assessed the effect of dyspeptic symptoms on eating, other daily activities, social contacts, sleep, and fears of serious disease. Finally, the patient's time spent off work and/or in hospital was recorded.
DATA MANAGEMENT AND STATISTICS

Data management was performed according to Good Clinical Practice and data were transferred to an independent statistical institute (Institut für numerische Statistik, Cologne, Germany). The treatment code was broken after decisions on the allocation of individual patients to intention to treat (ITT) and per protocol (PP) analyses. All analyses were based on SAS 11 and SPSS7.5 (for Windows).

The response rate based on the primary outcome variable was estimated to be $60 \%$ after placebo and $80 \%$ after omeprazole therapy. Thus accepting a $\beta$ error of 0.20 , the necessary number of patients per group in an ITT analysis would be 75 (Fisher's exact test, two sided), with an $\alpha$ error adjusted to 0.017 according to Bonferroni to compensate for comparisons between the three active treatment groups and placebo. This number was increased to 90 per group to provide for a valid PP analysis. The primary response rate was tested for centre effects using the Breslow-Day test.

Secondary outcome measures included effects of treatment on gastrointestinal symptoms and on QoL. These analyses were exploratory and used standard significance levels $(p<0.05)$. A life table analysis of relapses during the six month follow up period was performed.

POSSIBLE PROGNOSTIC FACTORS

The following factors were included in a logistic regression analysis using the main outcome criterion as the dependent variable: treatment, age, gender, smoking, alcohol consumption, dyspepsia, gastric erosions present, and positive urea breath test.

\section{Results}

PATIENTS

A flow chart showing the fate of the 974 patients entering the one week run in period is shown in fig 1 . The response rate to low dose antacid was $13 \%$ and was independent of the predominant symptom recorded at entry. Patients who were found to have a cause for their symptoms other than functional dyspepsia were excluded. The remaining 792 patients were symptomatic for at least three days of the run in week and were considered to require treatment. $H$ pylori positive and negative patients (422 and 370, respectively) were randomised to study treatments and data from those patients were included in an ITT analysis. PP analysis was performed on data from 354 and 315 patients (reasons for exclusion of 123 patients are summarised in table 1). There was a similar distribution of patient characteristics across the four treatment groups in both $H$ pylori positive and negative cohorts (table 2). Comparing the cohorts, $H$ pylori positive patients were older and more frequently male than $H$ pylori negative patients.

EFFECT OF TWO WEEK ANTISECRETORY TREATMENT

Patient response rates (ITT) at the end of two weeks of active or placebo treatment are 
Table 1 Reasons for exclusion of patients in the placebo, ranitidine $150 \mathrm{mg}$, omeprazole $10 \mathrm{mg}$ (OM10), and omeprazole 20 mg (OM20) groups from per protocol (PP) analyses

\begin{tabular}{|c|c|c|c|c|c|c|c|c|c|c|c|c|}
\hline \multirow[b]{2}{*}{ Patients randomised and included ITT (n) } & \multicolumn{3}{|c|}{ Placebo } & \multicolumn{3}{|c|}{ Ranitidine } & \multicolumn{3}{|c|}{ OM10 } & \multicolumn{3}{|c|}{ OM20 } \\
\hline & 203 & 113 & 90 & 194 & 111 & 83 & 202 & 95 & 107 & 193 & 103 & 90 \\
\hline Excluded PP & All & $\mathrm{H}_{p+}$ & $H_{p-}$ & All & $H p+$ & $H_{p-}$ & All & $H p+$ & $H_{p-}$ & All & $H p+$ & $H_{p}-$ \\
\hline Major deviation from inclusion/exclusion criteria & 2 & 1 & 1 & 0 & 0 & 0 & 2 & 1 & 1 & 1 & 0 & 1 \\
\hline Early termination due to $\mathrm{AE}$ & 1 & 1 & 0 & 1 & 1 & 0 & 1 & 1 & 0 & 1 & 1 & 0 \\
\hline Prohibited concomitant medication & 6 & 5 & 1 & 5 & 3 & 2 & 4 & 1 & 3 & 4 & 2 & 2 \\
\hline$<75 \%$ compliance with study treatment & 5 & 3 & 2 & 5 & 3 & 2 & 3 & 2 & 1 & 6 & 4 & 2 \\
\hline Non-compliance with visits & 13 & 6 & 7 & 15 & 9 & 6 & 11 & 6 & 5 & 17 & 10 & 7 \\
\hline Other major protocol violations & 5 & 2 & 3 & 6 & 2 & 4 & 5 & 1 & 4 & 4 & 3 & 1 \\
\hline Total exclusions & 32 & 18 & 14 & 32 & 18 & 14 & 26 & 12 & 14 & 33 & 20 & 13 \\
\hline Total included PP & 171 & 95 & 76 & 162 & 93 & 69 & 176 & 83 & 93 & 160 & 83 & 77 \\
\hline
\end{tabular}

ITT, intention to treat; AE, adverse effect.

Table 2 Pretreatment patient characteristics (intention to treat analysis) in the placebo, ranitidine $150 \mathrm{mg}$, omeprazole 10 $\mathrm{mg}$ (OM10), and omeprazole $20 \mathrm{mg}$ (OM20) groups

\begin{tabular}{|c|c|c|c|c|c|c|c|c|c|}
\hline & \multicolumn{4}{|c|}{ H pylori positive $(n=422)$} & \multicolumn{4}{|c|}{ H pylori negative $(n=370)$} & \multirow[b]{2}{*}{$p$ Value $^{a}$} \\
\hline & $\begin{array}{l}\text { Placebo } \\
(n=113)\end{array}$ & $\begin{array}{l}\text { Ranitidine } \\
(n=111)\end{array}$ & $\begin{array}{l}\text { OM10 } \\
(n=95)\end{array}$ & $\begin{array}{l}\text { OM20 } \\
(n=103)\end{array}$ & $\begin{array}{c}\text { Placebo } \\
(n=90)\end{array}$ & $\begin{array}{l}\text { Ranitidine } \\
(n=83)\end{array}$ & $\begin{array}{l}\text { OM10 } \\
(n=107)\end{array}$ & $\begin{array}{l}\text { OM20 } \\
(n=90)\end{array}$ & \\
\hline Mean age (y) & 46 & 48 & 47 & 46 & 40 & 38 & 41 & 43 & 0.00000 \\
\hline Sex (\% male) & 43 & 40 & 46 & 41 & 33 & 40 & 35 & 33 & 0.041 \\
\hline Cigarette smoking $^{\mathrm{b}}(\%)$ & 50 & 44 & 40 & 40 & 38 & 43 & 44 & 44 & 0.77 \\
\hline $\mathrm{Alcohol}^{\mathrm{b}}(\%)$ & 54 & 51 & 53 & 59 & 56 & 57 & 50 & 56 & 0.94 \\
\hline Dyspepsia $>5$ y $(\%)$ & 39 & 38 & 38 & 40 & 38 & 33 & 27 & 36 & 0.10 \\
\hline
\end{tabular}

${ }^{a}$ All $H$ pylori positive $v$ all $H$ pylori negative patients.

Includes patients who recently stopped consumption.

illustrated in fig 2 for the two outcomes: "no need for management" and "no dyspeptic symptoms". For comparisons, Fisher's exact test was used. Compared with placebo, a significant therapeutic gain was obtained only with OM20, the beneficial effect being both in terms of management $(17.6 \% ; \mathrm{p}=0.014$ using the Bonferroni adjusted $p$ level of 0.017 ) and abolition of symptoms $(19.6 \% ; \mathrm{p}=0.0005)$ in the $H$ pylori positive group. In the $H$ pylori negative group, there were no significant changes in the primary response (need for management) compared with placebo but in significantly more patients symptoms were abolished after OM20 compared with placebo $(16.7 \% ; \mathrm{p}=0.023)$. All changes (compared with placebo) are presented in table 3 and indicate that treatment with OM20 produced the greatest therapeutic benefit, especially in the $H$ pylori positive group. All treatments were more effective at reducing the intensity of dyspeptic symptoms below the "need for treatment" threshold than in abolishing symptoms altogether (fig 2). The results of the ITT

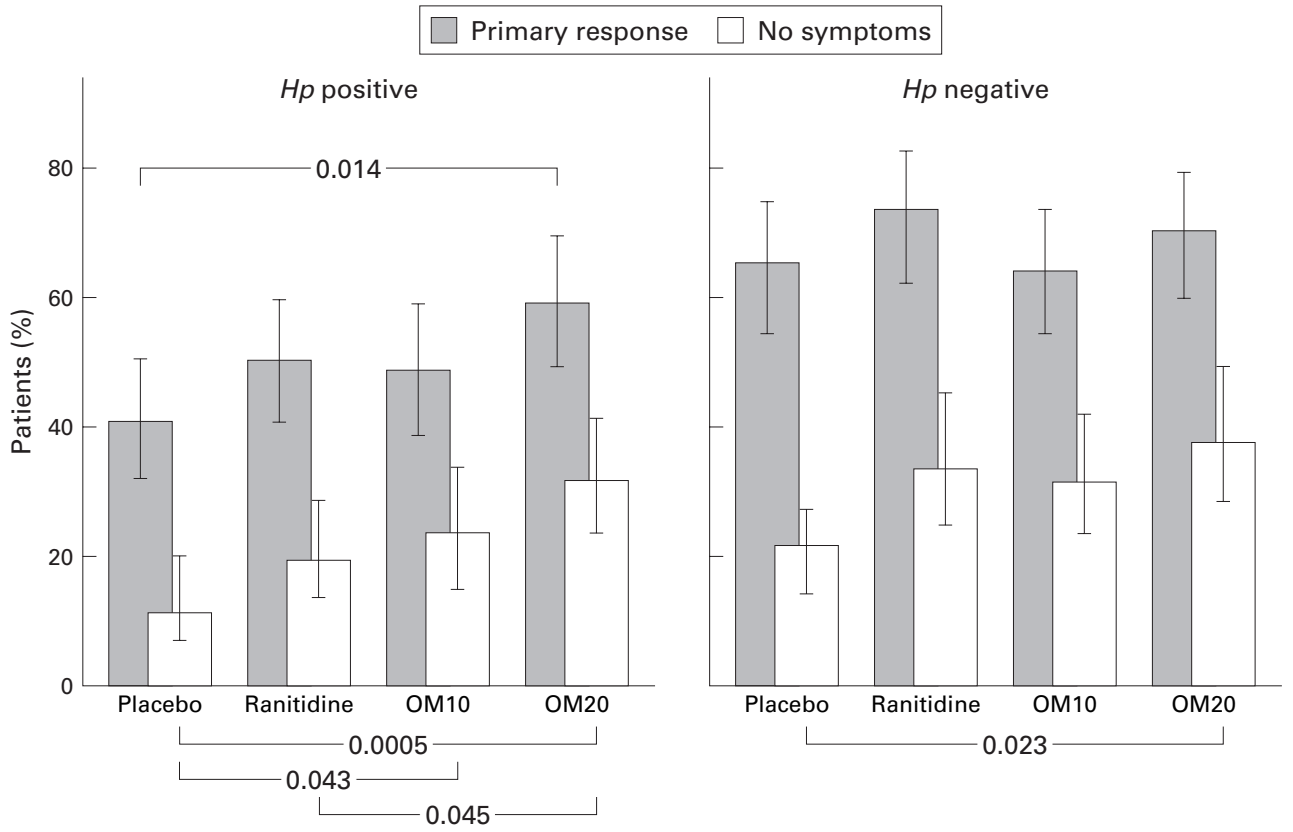

Figure 2 Effect of two week treatment with placebo, ranitidine, omeprazole $10 \mathrm{mg}$ (OM10), or omeprazole $20 \mathrm{mg}$ (OM20) on dyspeptic symptoms according to percentage of patients who became completely symptom free (no symptoms) and those with no need for further management (main outcome criterion) (means and 95\% two sided confidence intervals; exact test). For assessment of significance levels, see text. The number of patients per group is given in table 1. Hp, Helicobacter pylori. 
Table 3 Differences in response rates (per cent of patients with 95\% confidence intervals) at the end of the two week treatment period compared with placebo (therapeutic gain) in the ranitidine $150 \mathrm{mg}$, omeprazole $10 \mathrm{mg}$ (OM10), and omeprazole $20 \mathrm{mg}$ (OM20) groups.

"Primary response" indicates no need for management of dyspepsia; "No symptoms" indicates disappearance of all dyspeptic symptoms (intention to treat analysis)

\begin{tabular}{lrrr}
\hline & Ranitidine & \multicolumn{1}{l}{ OM10 } & OM20 \\
\hline H pylori positive & & & \\
$\quad$ Primary response (\%) & $8.9(-4.2-21.9)$ & $6.8(-6.7-20.4)$ & $17.6(4.2-31.0)^{\star \star}$ \\
$\quad$ No symptoms (\%) & $7.4(-2.2-17.1)$ & $10.8(0.4-21.1)^{\star}$ & $19.6(8.6-30.7)^{\star \star \star} \dagger$ \\
H pylori negative & & & \\
Primary response (\%) & $7.9(-5.8-21.7)$ & $-1.1(-14.4-12.3)$ & $5.5(-8.0-19.1)$ \\
$\quad$ No symptoms (\%) & $12.7(-0.7-26.2)$ & $10.5(-2.1-23.1)$ & $16.7(3.2-30.1)^{\star \star}$ \\
\hline
\end{tabular}

${ }^{\star} \mathrm{p}<0.05,{ }^{\star \star} \mathrm{p}<0.025,{ }^{\star \star \star} \mathrm{p}<0.001$ compared with placebo; $t \mathrm{p}<0.05$ compared with ranitidine in the same $H$ pylori group. All other differences within the same $H$ pylori group were not significant $(\mathrm{p}>0.05)$.
With the other treatments, no significant differences from placebo were found for either $H$ pylori positive or negative patients.

ADVERSE EVENTS

The adverse events noted during the study are described below for the four treatment groups and numbers in parentheses refer to $H$ pylori positive and $H$ pylori negative status, respectively. Eight patients (placebo $3(2 / 1)$; ranitidine 4 (3/1); OM10 $1(0 / 1)$ ) stopped treatment early because of worsening symptoms (they were included in the PP analyses as "treatment failures") and three patients because of other adverse events: ranitidine $1(1 / 0)$ vascular disorder; OM10 1 (1/0) taste disorder; OM20 $1(1 / 0)$ scarlet fever (these three patients were excluded from PP analyses). One OM20 (1/0) patient who developed borreliosis completed the study. All other adverse events (placebo 21 (11/10); ranitidine 28 (14/14); OM10 28 (12/16); OM20 26 (14/11)) were not serious and had no effect on the course of the study or analyses. patients in each group. OM20 significantly reduced all three symptoms compared with placebo in the $H$ pylori positive patients but only epigastric pressure/fullness was significantly reduced in $H$ pylori negative patients by all three active treatments.

QoL parameters and impairment caused by dyspepsia were analysed in $H$ pylori positive and negative cohorts before and after treatment. Baseline QoL was similar across the treatment groups for both $H$ pylori positive and negative cohorts and there was significant improvement within all groups (including placebo) following treatment (Wilcoxon signed rank test). However, when improvement was compared between treatments, OM20 improved QoL to a significantly greater extent than placebo on seven out of nine parameters measured in $H$ pylori positive patients (Wilcoxon rank sum test). In $H$ pylori negative patients, OM20 was significantly better than placebo on only one of nine items of the QoL.
LOGISTIC REGRESSION ANALYSIS OF FACTORS AFFECTING THE PRIMARY OUTCOME RESPONSE In a logistic regression analysis of data from all patients $(n=792)$ using the factors previously described, antisecretory treatment and $H$ pylori infection had a statistically significant influence on treatment outcome. The odds ratios for the two factors were 1.39 (95\% CI 1.00-1.93) and 0.46 (95\% CI 0.34-0.62), respectively. When, instead of "antisecretory treatment", each of the four treatment groups was entered separately into the logistic regression analysis, the only statistically significant factor was $H$ pylori infection. When $H$ pylori positive and negative patients were analysed separately, excluding $H$ pylori status from the analysis, a significant influence of antisecretory treatment was observed only in $H$ pylori infected patients (odds ratio $1.57,95 \%$ CI $1.01-2.42$ ). No other factors proved to be significant in either group.

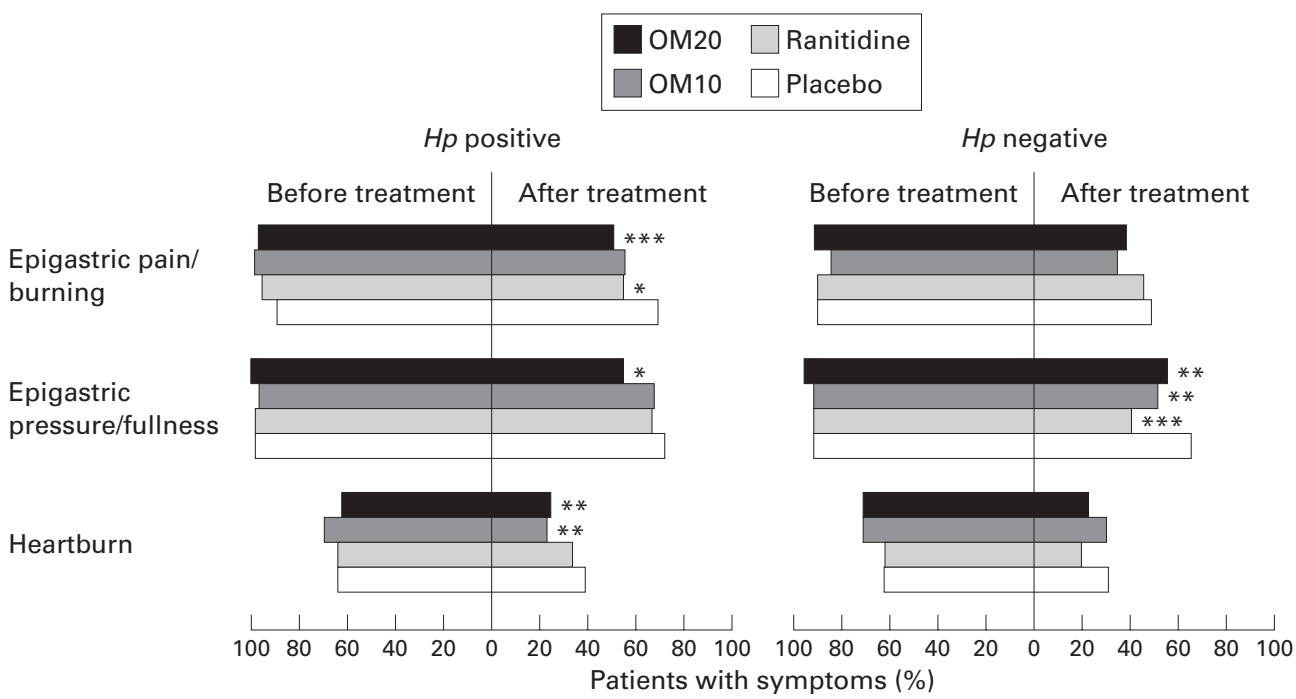

Figure 3 Effect of treatment with placebo, ranitidine, omeprazole $10 \mathrm{mg}$ (OM10), or omeprazole $20 \mathrm{mg}$ (OM20) on individual dyspeptic symptoms. The horizontal bars show the percentages of patients with each individual symptom before and after each two week double blind treatment. ${ }^{\star} p<0.05 ;{ }^{\star} p<0.025,{ }^{\star}{ }^{\star} p<0.001$ compared with placebo in the same Helicobacter pylori $(H P)$ group. 


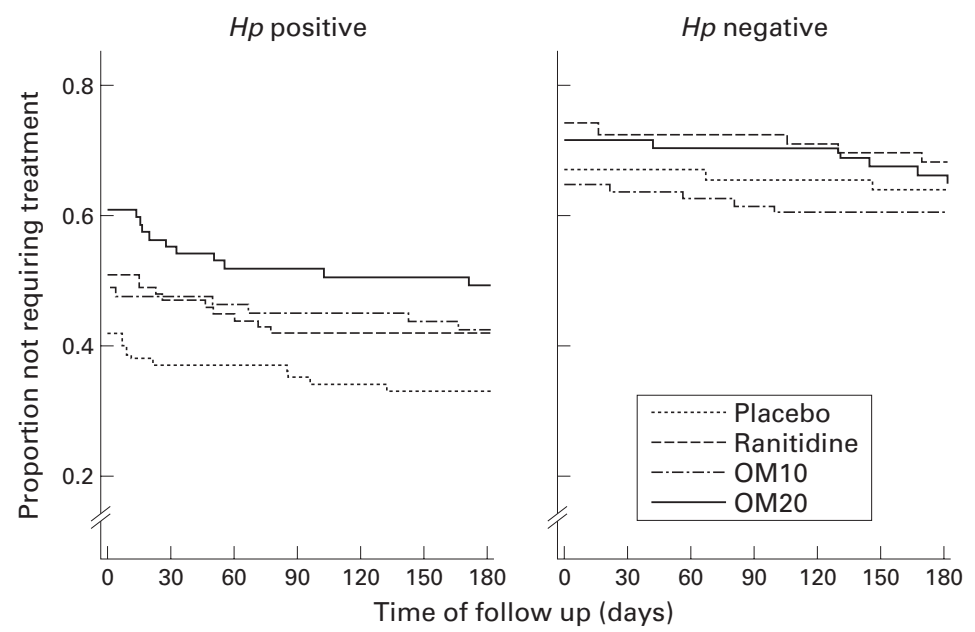

Figure 4 Life table analysis of patients without sufficient symptoms to require specific active management after successful treatment in the four treatment groups (placebo, ranitidine, omeprazole $10 \mathrm{mg}$ (OM10), or omeprazole $20 \mathrm{mg}$ (OM20)). At time 0 the proportion of patients with successful two week treatment is given; these patients entered follow up. The number of patients per group is given in the text. Hp, Helicobacter pylori.

RESULTS OF FOLLOW UP

A total of 427 patients (placebo $n=97$, ranitidine $\mathrm{n}=108, \mathrm{OM} 10 \mathrm{n}=106, \mathrm{OM} 20$ $\mathrm{n}=116$ ) entered follow up. Forty nine per cent of patients remained symptom free and $34 \%$ had minor symptoms below the need for treatment threshold during the six month follow up period. Only $18 \%$ of patients (25\% of the $H$ pylori positive group and $12 \%$ of the $H$ pylori negative group) had a relapse as defined by the protocol-that is, reappearance of symptoms requiring management. Thirty four patients (total followed by $H$ pylori positive/H pylori negative: placebo $6(6 / 0)$; ranitidine $10(8 / 2)$; OM10 7 (5/2); and OM20 11 (7/4)) were re-endoscoped. Two (2/0) ranitidine patients had erosive duodenitis; all others had normal endoscopic findings. Life table analysis of symptomatic relapse is shown in fig 4 . The symptom pattern of relapses closely resembled that observed on admission to the study. Interestingly, OM20 was the most effective treatment for the prevention of relapse in the $H$ pylori positive group while relapse rates were similar with OM20 and ranitidine $150 \mathrm{mg}$ (and not significantly different from placebo) in the $H$ pylori negative group.

\section{Discussion}

In this large controlled clinical trial, the primary outcome criterion was disappearance of dyspeptic symptoms requiring further management after a two week treatment course. Using this main criterion, which reflects the treatment of dyspeptic patients in general practice, we observed a favourable effect of omeprazole, given at a dose of $20 \mathrm{mg}$ per day, in the treatment of dyspeptic patients infected with $H$ pylori. Conversely, no significant favourable effect was observed in non-infected dyspeptic patients. Using the same outcome criterion, a lower dose of omeprazole, $10 \mathrm{mg}$ a day, and ranitidine given at a dose presently recommended for over the counter treatment of dyspeptic patients, ${ }^{4}$ were ineffective both in infected and non-infected patients. A favour- able effect of omeprazole and ranitidine in non-infected patients cannot be totally excluded because a secondary outcome criterion (that is, complete disappearance of symptoms) appeared to be favourably affected by these two drugs. However, as the primary outcome criterion remained unchanged, this observation is of little importance.

The primary effect of omeprazole $20 \mathrm{mg}$, albeit statistically significant, was weak. The therapeutic gain over placebo observed in $H$ pylori positive subjects was only $17 \%$ and was lower than the actual placebo response (42\%) in the same group of patients. The therapeutic gain was similar $(20 \%)$ when, instead of the primary outcome criterion, complete disappearance of dyspeptic symptoms was used. Even with $20 \mathrm{mg}$ of omeprazole, only about one third of patients became asymptomatic during the two week treatment period.

The two week treatment period was chosen in the present study because in previous positive studies a favourable drug effect was observed within two weeks. ${ }^{11}$ We cannot exclude the possibility that prolongation of treatment may further improve the favourable effect of $20 \mathrm{mg}$ of omeprazole. However, it may, in parallel, also increase the placebo effect, which appears to increase over time. ${ }^{11}$ In the present study, low dose antacid treatment, which in previous studies was not different from placebo treatment, ${ }^{11}$ was given for one week before double blind treatment was started and was successful in $13 \%$ of individuals treated. In subjects who did not respond to low dose antacids, the effectiveness of a two week placebo treatment was considerable. This placebo effect might be due in part to the relief of obtaining negative endoscopy results ${ }^{12}$ and also to the natural history of functional dyspepsia, which typically produces selflimiting symptomatic episodes followed by prolonged periods with little or no symptoms. ${ }^{13}$ This observation was confirmed during the follow up period of the present study (see fig 4).

The decision was taken not to blind the investigators to $H$ pylori status because in Germany a large portion of dyspeptic patients already know their $H$ pylori status. Furthermore, it was planned to give antibiotic treatment to $H$ pylori positive patients who did not respond to antisecretory treatment. Again, this procedure was intended to resemble, as much as possible, rountine treatment of dyspeptic patients in clinical practice. The study investigators were instructed to include $H$ pylori positive non-responders in a second double blind trial. This option, not available to $H$ pylori negative subjects, could theoretically have led to some bias in the investigators, by making them more prone to recognise therapeutic failure in $H$ pylori positive than in $H$ pylori negative patients. This bias, rather than a more severe form of the disease, may have been responsible for the lower global responsiveness to treatment, including placebo, in $H$ pylori positive than in $H$ pylori negative subjects. However, due to the double blind administration of study treatment, such a bias cannot 
explain the finding that antisecretory treatment with OM20 had a significant beneficial effect in $H$ pylori positive patients while in general it was considerably less effective in $H$ pylori negative subjects. Therefore, we conclude that OM20 is more effective in $H$ pylori positive subjects.

The higher therapeutic gain in response to $20 \mathrm{mg}$ of omeprazole in $H$ pylori positive subjects compared with negative subjects might be due to the effect of omeprazole on $H$ pylori. ${ }^{14}$ Omeprazole causes overall suppression of Helicobacter growth in the stomach and redistribution of inflammation, which improves in the antrum and increases in the corpus. Lower antral inflammation may theoretically normalise gastrin levels, antral motility, and visceral sensitivity, and thus improve functional dyspepsia. ${ }^{15}$ However, more aggressive Helicobacter treatment hardly improved the symptoms of functional dyspeptics in several controlled clinical trials. ${ }^{16-19}$ Also, in our $H$ pylori positive patients, Helicobacter treatment did not have a favourable effect, either in those patients with an inadequate primary response to treatment or in those who first responded and later had a recurrence..$^{20}$ Thus we conclude that neither suppression of $H$ pylori nor associated improvement of gastritis is responsible for the favourable effect of omeprazole. An alternative mechanism is improvement in $\mathrm{pH}$ control by omeprazole in the presence of concomitant $H$ pylori infection ${ }^{6}$ which could, in turn, improve acid induced symptoms of dyspeptics. ${ }^{21}$ Finally, acid may play a more important role in infected subjects than in non-infected subjects; the infected mucosa could be more acid sensitive than the non-infected mucosa, or duodenal acid clearance could be slower in infected than in non-infected subjects. ${ }^{1521}$ Functional dyspepsia provides yet another example of where a better clinical effect with proton pump inhibitors is found in $H$ pylori infected subjects. Previous examples include treatment and prevention of recurrence of reflux oesophagitis ${ }^{22}$ as well as treatment of ulcers induced by nonsteroidal anti-inflammatory drugs. ${ }^{23}$

It could be argued that the difference between $H$ pylori positive and $H$ pylori negative subjects was caused by an unequal distribution of the two collectives. In fact, as expected, ${ }^{24}$ infected dyspeptics were older than noninfected subjects in our study and were more frequently male. These two characteristics, age and sex, might be associated with a better response of acid symptoms to antisecretory drugs. ${ }^{25-27}$ To assess this possibility, we performed a logistic regression analysis using all possible confounders. We found that $H$ pylori infection was the only factor associated with outcome when all dyspeptic patients were analysed together. In the group of subjects with $H$ pylori infection, the only factor associated with outcome was antisecretory treatment, while in non-infected subjects no predictor was identified in our logistic regression analysis. Therefore, unequal distribution of age and sex cannot explain the different responses of infected and non-infected dyspeptics to antisecretory treatment.
In this study we included patients with heartburn provided they also had epigastric symptoms. We excluded patients with reflux symptoms alone. In these patients, reflux disease appeared to be likely while in patients admitted with heartburn and epigastric symptoms, it remained a possibility, in spite of a normal endoscopic appearance of the oesophagus. ${ }^{28}$ Oesophageal pHmetry, which is thought by some authors to represent the gold standard for the diagnosis of reflux disease, ${ }^{29}$ was not feasible in this large multicentre trial and may not in fact have been helpful as it may give abnormal results in asymptomatic subjects and in those with functional dyspepsia who do not complain of heartburn. ${ }^{30}$ Conversely, heartburn patients may have normal pHmetry. ${ }^{31}$ Patients with a sensitive oesophagus suffer from acid induced symptoms in spite of normal pHmetry. ${ }^{32}$ In others, the cause of heartburn is not clear as they have normal pHmetry and do not respond to high dose antisecretory treatment. ${ }^{33}$ We noted that patients with and without heartburn showed similar responses to treatment and omeprazole improved epigastric pain and pressure as well as heartburn. These observations make it highly unlikely that omeprazole has a favourable effect on functional dyspepsia simply because it improves reflux symptoms. Our study argues against excluding heartburn patients who also complain of epigastric symptoms from controlled clinical trials and from definitions of functional dyspepsia.

We were surprised by the low recurrence rate of dyspeptic symptoms during the six month follow up period. The higher recurrence rate in $H$ pylori positive patients was probably a consequence of biased assessment by the investigators, as was the case for the higher placebo response. More importantly, there was no evidence of a higher recurrence rate after omeprazole in spite of reports of rebound acid secretion in $H$ pylori negative patients after omeprazole treatment. ${ }^{34}$

Recently, the question has been raised as to whether or not $H$ pylori infection should be treated in functional dyspeptics. ${ }^{17}$ On the basis of this study, proton pump inhibitors may be less effective after cure of infection. However, this needs to be tested in another trial but for now it is yet another argument against $\mathrm{H}$ pylori treatment in functional dyspepsia.

In conclusion, omeprazole $20 \mathrm{mg}$ per day improved dyspeptic symptoms in patients infected with $H$ pylori to a greater extent than in those who were $H$ pylori negative. The effect was relatively weak however, and identification of those patients who are likely to respond requires additional studies.

We are grateful to Dr Madeline Frame for expert assistance with the manuscript preparation. Supported by Swiss Science Research Foundation, grant No 31-43240.95, and by AstraHässle, AB, Sweden.

\section{Appendix}

FROSCH STUDY GROUP

Werner Abels (Nürnberg), Krikor Amdja (Wermelskirchen), Walter Armknecht (Worms), Amelie Arnold (Marburg), Herbert Bock 
(Frankfurt), Dibor Bojanovski (Hannover), Hilmar Böneke (Lienen), Hubert Brinkhoff (Stuhr), Burkhardt Cyrus (München), Axel Dettmer (München), Reinhard Diedrich (Marburg), Klaus Dietrich (Saarbrücken), Bernhard Dirr (Hechingen), Wolfgang Dübel (Berlin), Michael Dudek (Düsseldorf), Dieter Ebbinghaus (Lünen), Horst Feyerabend (Hagen), Wolfgang Fortelny (Waldsassen), Frank Fueting (Nassau), Meinolf Golsong (Oberhausen), Roland Graf (Leutkirch), Wolfgang Güttel (Rastatt), Hans-Jürgen Hagel (Schwabach), Bernt Hawickhorst (Wülfrath), Martin Hell (Salzgitter-Lebenstedt), Rüdiger Hildebrandt (Clausthal-Zellerfeld), Gert-Heintz Hirschmann (Berlin), Andreas Hoffmann (Magdeburg), Walter Hofmeister (Weiden), Hermann Hornstein (Pfullingen), G. Hornung (Essen), Wolfgang Huppertz (Essen), Harvey Juergens (Ölde), Bernhard Klesser (Ulm), Horst Klewer (Wetzlar), Michael Klöters (Spaichingen), Andreas Kocjan (Lüdenscheid), Justine Kosmowski (Frankfurt), Thomas Krummenerl (Münster), Joachim Labenz (Essen), Günter Leiber (Marburg), Christian Leischik (Köln), Helmut Lichti (Gladenbach), Albin Lütke (Koblenz), Andrei Mares (Frankfurt), Eckard Massmann (Münster), Peter Mayr (Stockach), Bernd Metscher (Berlin), Heinrich Miks (Hamm), Hubert Mönnikes (Marburg), Michael Müller (Delmenhorst), Claus Nolte (Mettmann), Paul-Peter Pech (Münster), Wilhelm Peters (Duisburg), Stefan Pfäffl (Nürnberg), Thomas Rachel (Rastatt), Axel Rambow (Frankfurt), Gerd Rosprich (Saarbrücken), Thomas Schädlich (Ellefeld), Jost Schattenberg (Wiesbaden), Dieter Schlauch (Ludwigshafen), Volker Schmid (Fürstenzell), Andreas Schober (Göttingen), Michael Schumacher (Wolmirstedt), Erich Schütz (Regensburg), Rainer Stroband (Münster), Gerard Tangerding (Wangen), Peter Tippmann (Esslingen), Huschang Toluipur (Schiffweiler), Rüdiger Vogt (Mannheim), Rainer Wack (Berlin), Hannelore Wiechmann (Potsdam), Günter Wilhelms (Goslar), Friedrich Winter (Burgebrach), Jürgen Zeus (Erlangen).

1 Talley NJ, Meineche-Schmidt V, Paré P, et al. Efficacy of omeprazole in functional dyspepsia: double-blind, rand-
omized, placebo-controlled trials (the Bond and Opera omized, placebo-controlled trials (the Bond and
studies). Aliment Pharmacol Ther 1998:12:1055-65.

2 Meineche-Schmidt V, Christensen E. Classification of dyspepsia. Identification of independent symptom components in 7270 consecutive, unselected dyspepsia patients from general practice. Scand $\mathcal{F}$ Gastroenterol 1998;33:126272.

3 Talley NJ ed. Dyspepsia. Baillière's Clinical Gastroenterology vol. 12/No 3. London: Baillière Tindall, 1998.

4 Pappa KA, Gooch WM, Buaron K, et al. Low-dose ranitidine for the relief of heartburn. Aliment Pharmacol Ther 1999;13:459-66.

5 Agreus L, Talley NJ. Dyspepsia: current understanding and management. Annu Rev Med 1998;49:475-93.

6 Verdú EF, Armstrong D, Idström JP, et al. Intragastric pH during treatment with omeprazole: role of Helicobacter pylori and H. pylori-associated gastritis. Scand $\mathcal{F}$ Gastroenpylori and $H$. pylori-as
terol $1996 ; 31: 1151-6$.

7 Price AB. The Sydney system: Histological division. 7 Gastroenterol Hepatol 1991;6:209-22.
8 Labenz J, Börsch G, Peitz U, et al. Validity of a novel biopsy urease test (HUT) and a simplified ${ }^{13} \mathrm{C}$-urea breath test for diagnosis of Helicobacter pylori infection and estimation of the severity of gastritis. Digestion 1996;57:391-7.

9 Siegrist J, Middeke M, Osterkorn K. Lebensqualität hypertensiver Ärzte unter Hochdrucktherapie. Fortschr Med 1991;109:348-52

10 Eypasch E, Wood-Dauphinée S, Williams JI, et al. Der gastrointestinale Lebensqualitätindex (GLQI). Ein klinimetrischer Index zur Befindlichkeitsmessung in der gastroenterologischen Chirurgie. Chirurg 1993;64:264-74.

11 Holtmann G, Talley NJ. Functional dyspepsia. Current treatment recommendations. Drugs 1993;45:918-30.

12 Bytzer P, Hansen JM, Schaffalitzky de Muckadell OB. Empirical H2-blocker therapy or prompt endoscopy in management of dyspepsia. Lancet 1994;343:811-16.

13 Locke GR, III. Prevalence, incidence and natural history of dyspepsia and functional dyspepsia. In: Talley NJ, ed. Balliere's Clinical Gastroenterology, volume 12, No 3. London: Ballière Tindall, 1998;435-42.

14 Stolte M, Meining A, Schmitz JM, et al.. Changes in Helicobacter pylori-induced gastritis in the antrum and corpus during 12 months of treatment with omeprazole and lansoprazole in patients with gastro-oesophageal reflux disease. Aliment Pharmacol Ther 1998;12:247-53.

15 Pantoflickova D, Blum AL, Koelz HR. Helicobacter pylori and functional dyspepsia: a real causal link? In: Talley NJ, ed. Ballière's Clinical Gastroenterology, volume 12, No 3. London: Ballière Tindall, 1998;503-32.

16 Blum AL, Talley NJ, O'Morain O, et al. Lack of effect of treating Helicobacter pylori infection in patients with nonulcer dyspepsia. N Engl f Med 1998;339:1875-81.

17 Friedman LS. Helicobacter pylori and nonulcer dyspepsia. $N$ Engl F Med 1998;339:1928-30.

18 McColl K, Murray L, El-Omar, et al. Symptomatic benefit from eradicating Helicobacter pylori infection in patients with nonulcer dyspepsia. $N$ Eng $\mathcal{F}$ Med 1998;339:1869-74.

19 Talley NJ, Janssens J, Lauritsen K, et al. Eradication of Helicobacter pylori in functional dyspepsia: randomised Helicobacter pylori in functional dyspepsia: randomised follow-up. $B M \mathfrak{F} 1999 ; 318: 833-7$

20 Koelz HR, Arnold R, Stolte M, et al. Treatment of Helicobacter pylori in functional dyspepsia resistant to conventional management. A 6-month controlled assessment. Gut 2000 (in press).

21 Samson M, Verhagen MAMT, van Berge Henegouwen GP, et al. Abnormal clearance of exogenous acid and increased acid sensitivity of the proximal duodenum in dyspeptic patients. Gastroenterology 1999;116:515-20.

22 Carlsson R. Gastroesophageal reflux disease. A study of pathophysiology, clinical diagnosis and management. Thesis. Göteborg: University Press, 1998.

23 Hawkey CJ, Tullassay Z, Szepaski E, et al. Failure of Helicobacter pylori eradication to influence site-specific relapse in high risk NSAID users: results of a large six month double blind randomised controlled clinical trial. Gastroenterology 1998;114:A145 (GO594).

24 Thümer HL, Flaaten B, Erichsen KE, et al. Helicobacter pylori infection in non-ulcer dyspeptic and ulcer patients. Determinants and consequences in 'real life'. Scand $\mathcal{F}$ Gastroenterol 1996;31:744-8.

25 Bennet EJ, Plesse C, Palmer K, et al. Functional gastrointestinal disorders: psychological, social, and somatic features. Gut 1998;42:414-20.

26 Finney JS, Kinnersley N, Hughes M, et al. Meta-analysis of antisecretory and gastrokinetic compounds in functional dyspepsia. f Clin Gastroenterol 1998;26:312-20.

27 Herschbach P, Henrich G, von Rad M. Psychological factors in functional gastrointestinal disorders: characteristics of the disorder or of the illness behavior? Psychosom Med 1999;61:148-53.

28 Klauser AG, Voderholzer WA, Knesewitsch PA, et al. What is behind dyspepsia? Dig Dis Sci 1993;38:147-54

29 Klauser AG, Schindlbeck NE, Müller-Lissner SA. SympKlauser AG, Schindlbeck NE, Müller-Lissner SA. Symp-
toms in gastro-eosophageal reflux disease. Lancet 1990;335: 205-8.

30 Pfeiffer A, Aronbayev J, Schmidt T, et al. Gastric emptying, esophageal 24-hour $\mathrm{pH}$ and gastric potential difference measurements in non-ulcer dyspepsia. Gastroenterol Clin Biol 1992;16:395-400.

31 Lind T, Havelund T, Carlsson R, et al. Heartburn without oesophagitis: efficacy of omeprazole therapy and features determining therapeutic response. Scand f Gastroenterol 1997;32:974-9.

32 Smout AJPM. Evaluation and significance of reflux from the duodenum and stomach. Aliment Pharmacol Ther 1997; 11(suppl 2):27-32.

33 Schenk BE, Kuipers EJ, Klinkenberg-Knol EC, et al. Omeprazole as a diagnostic tool in gastroesophageal reflux disprazole as a diagnostic tool in gastroesophage

34 Gillen D, Wirz AA, Ardill JE, et al. Rebound hypersecretion after omeprazole and its relation to on-treatment acid suppression and Helicobacter pylori status. Gastroenterology 1999;116:239-47. 